\begin{tabular}{|r|l|}
\hline \multicolumn{2}{|c|}{ Statistica Sinica Preprint No: SS-2021-0140 } \\
\hline Title & $\begin{array}{l}\text { Sparse and Low-Rank Matrix Quantile Estimation With } \\
\text { Application to Quadratic Regression }\end{array}$ \\
\hline Manuscript ID & SS-2021-0140 \\
\hline URL & http://www.stat.sinica.edu.tw/statistica/ \\
\hline DOI & $10.5705 /$ ss.202021.0140 \\
\hline Complete List of Authors & $\begin{array}{l}\text { Wenqi Lu, } \\
\text { Zhongyi Zhu and } \\
\\
\text { Heng Lian }\end{array}$ \\
\hline Corresponding Author & Heng Lian \\
\hline E-mail & henglian@cityu.edu.hk \\
\hline Notice: Accepted version subject to English editing. \\
\hline
\end{tabular}


Statistica Sinica

\title{
SPARSE AND LOW-RANK MATRIX QUANTILE ESTIMATION WITH APPLICATION TO QUADRATIC REGRESSION
}

\author{
Wenqi Lu ${ }^{1,2}$, Zhongyi Zhu² and Heng Lian ${ }^{1,3}$ \\ City University of Hong Kong ${ }^{1}$ and Fudan University ${ }^{2}$ \\ and CityU Shenzhen Research Institute ${ }^{3}$
}

Abstract: This paper studies matrix quantile regression where the covariate is a matrix and the response is a scalar. Statistical estimation of matrix regression is an active field of research. However, quantile regression with matrix covariates is rarely studied. We propose an estimation procedure based on convex regularizations in the high-dimensional setting. In order to reduce the dimensionality, the coefficient matrix is assumed to be low-rank and/or sparse. Thus we impose two regularizers to encourage the different low-dimensional structures. The asymptotic properties and implementation based on incremental proximal gradient algorithm are developed. We then apply the proposed estimator to quadratic quantile regression. The advantages of the proposed method in its applications to quadratic regression are also illustrated by simulations and real data analysis.

Key words and phrases: Dual norm, Interaction effects, Matrix regression, Penalization. 


\section{Introduction}

Quantile regression (Koenker and Bassett, 1978) is a useful statistical tool in data analysis. It provides a complement to the mean regression, allowing us to analyze the entire conditional distribution by modelling the covariate effects at different quantile levels. Despite a large literature on theoretical and computational aspects of vector covariate quantile regression (Koenker, 2005; Belloni and Chernozhukov, 2011; Yu et al., 2017; Yi and Huang, 2017), matrix quantile regression is rarely studied. However, matrix data arise frequently in fields such as digital image analysis (Zhou and Li, 2014), multi-task regression (Yuan et al., 2007; Argyriou et al., 2008; Bunea et al., 2012), matrix completion Candes and Plan, 2010; Koltchinskii et al., 2011; Negahban and Wainwright, 2012) and quadratic regression (Bien et al. 2013).

The primary challenge in matrix data analysis is its typically highdimensional nature. The popular way to reduce the dimensionality is to impose a sparsity assumption on covariates which is often encouraged by penalties such as Lasso (Tibshirani, 1996), SCAD (Fan and Li, 2001), elastic net (Zou and Hastie, 2005) and many others. For high-dimensional vector quantile regression with sparsity assumptions, Belloni and Chernozhukov (2011) established an uniform convergence rate for $\ell_{1}$ penalization, and 
then Zheng et al. (2015) achieved the oracle rate by employing adaptive Lasso penalty. There are also many other recent works studying related problems, see for example Kato (2011); Chao et al. (2017); Belloni et al. (2019); Pan and Zhou (2020).

For matrix data, the low-dimensional structure can be in the form of not only sparsity but also low-rankness. Nuclear norm is a convex relaxation of matrix rank, so it is used as penalty in many penalized least squares approaches to encourage low rankness (Yuan et al., 2007; Argyriou et al., 2010; Koltchinskii et al., 2011; Negahban and Wainwright, 2011; Zhou and Li, 2014). Other penalties such as rank (Bunea et al., 2011), the von Neumann entropy (Koltchinskii, 2011), and Schatten-p norm (Rohde and Tsybakov, 2011) were also used. Furthermore, some works consider lowrankness combined with sparsity to further improve dimension reduction or interpretation. For example, Agarwal et al. (2012) decomposed the true signal into a sum of a low-rank matrix and a sparse matrix. There are also works assuming the coefficient matrix satisfying low-rankness and sparsity simultaneously, such as the sparse reduced-rank regression (Chen et al., 2012; Ma et al., 2014) and two-step joint rank and row selection estimator (Bunea et al., 2012). However, these works are all based on penalized least squares. 
We propose an estimator in quantile regression with matrix covariates and scalar response in a high-dimensional setting. Compared with mean regression, quantile regression has advantages in robustness to outliers, skewness and heterogeneity, and it can be used to build prediction intervals. In order to deal with high dimensionality, we apply convex regularization techniques. In particular, we assume the underlying matrix lies in a lowdimensional subspace which is both sparse and low-rank, and then provide a convex regularized optimization approach using both nuclear norm and entry-wise $\ell_{1}$ norm as regularizers to exploit the low-dimensional structure. Unlike some previous approaches, our method encourages low-rankness and sparsity simultaneously. Moreover, we derive the upper bound on the estimation error of the proposed method in the high-dimensional setting. Theoretical results for high-dimensional quantile regression is more complicated than the least squares regression models. It also requires more technical analysis associated with matrix norms compared with penalized quantile regression with vector coefficients.

We then apply the matrix quantile regression to linear quantile regression with interaction effects. Dimension reduction is desirable for models with interactions, because even when the number of covariates $p$ is moderate, quadratic regression involves $O\left(p^{2}\right)$ parameters. Several variable selec- 
tion methods have been proposed to reduce the number of parameters for quadratic regression, including regularization methods (Choi et al., 2010, Bien et al., 2013; Hao et al., 2018) and screening (Hao and Zhang, 2014, Fan et al., 2015). All these works rely on the sparsity assumption, which requires that the number of significant variables is small and the signal size is large enough. We consider an alternative strategy using matrix regression, which does not necessarily require sparsity. Note that by writing $\mathbf{Z}_{i}=\left(1, \mathbf{x}_{i}^{\top}\right)^{\top}\left(1, \mathbf{x}_{i}^{\top}\right)$ where $\mathbf{x}_{i}$ is a $p$-dimensional vector predictor, the main effect $\mathbf{x}_{i}$ and quadratic interactions are all incorporated in matrix form. Thus a rank constraint can be used to restrict the effective number of parameters.

The rest of the paper is organized as follows. In Section 2, we introduce the estimator for matrix quantile regression model based on regularization, and present the implementation details and application to quadratic regression. Section 3 establishes the theoretical properties. In Section 4 , we investigate the finite sample properties on simulated and real datasets in quadratic quantile regression. We conclude the paper in Section 5. 


\section{Matrix quantile regression}

\subsection{General model setup}

In this paper, we study a matrix quantile regression model with scalar response $y \in \mathbb{R}$ and matrix covariate $\mathbf{Z} \in \mathbb{R}^{d_{1} \times d_{2}}$. Define the $\tau$ th conditional quantile of $y$ given $\mathbf{Z}$ as $Q_{\tau}(y \mid \mathbf{Z})=\inf \left\{t: F_{y \mid \mathbf{Z}}(t) \geq \tau\right\}$, where $F_{y \mid \mathbf{Z}}(t)$ is the conditional distribution function. We consider the setting that, for a certain quantile level $\tau \in(0,1), Q_{\tau}(y \mid \mathbf{Z})$ is modeled by the linear regression model

$$
Q_{\tau}(y \mid \mathbf{Z})=\langle\mathbf{B}, \mathbf{Z}\rangle
$$

where $\mathbf{B} \in \mathbb{R}^{d_{1} \times d_{2}}$ and $\langle\mathbf{B}, \mathbf{Z}\rangle=\operatorname{tr}\left(\mathbf{B}^{\top} \mathbf{Z}\right)=\langle\operatorname{vec}(\mathbf{B})$, $\operatorname{vec}(\mathbf{Z})\rangle$ is the inner product between matrices. In the above, we omitted the intercept for simplicity, which does not play a significant role in developing the theory, but would certainly be useful in practice. On the other hand, we will see later that the intercept is already incorporated into $\mathbf{B}$ for quadratic regression and thus in such a special case an additional intercept in 2.1 is not necessary.

We apply the convex regularization framework to estimate coefficient $\mathbf{B}$ under low-dimensionality assumptions including low-rankness and sparsity. Given an i.i.d. sample $\left(y_{i}, \mathbf{Z}_{i}\right), i=1, \ldots, n$, the regularized estimator is 
defined by

$$
\widehat{\mathbf{B}}=\arg \min \frac{1}{n} \sum_{i=1}^{n} \rho_{\tau}\left(y_{i}-\left\langle\mathbf{B}, \mathbf{Z}_{i}\right\rangle\right)+\lambda_{1} \mathcal{R}_{1}(\mathbf{B})+\lambda_{2} \mathcal{R}_{2}(\mathbf{B})
$$

where $\rho_{\tau}(u)=u(\tau-I\{u<0\})$ is the check loss function, $\mathcal{R}_{1}(\mathbf{B})$ and $\mathcal{R}_{2}(\mathbf{B})$ are the regularizers to exploit low-rankness and sparsity structure, respectively. Let $\left(\sigma_{1}(\mathbf{B}), \ldots, \sigma_{r}(\mathbf{B})\right)$ be the nonzero singular values of $\mathbf{B}$, with $r=\operatorname{rank}(\mathbf{B})$ the rank of $\mathbf{B}$. The nuclear norm $\|\mathbf{B}\|_{*}=\sum_{j=1}^{r} \sigma_{j}(\mathbf{B})$ is a convex relaxation of $\operatorname{rank}(\mathbf{B})$. Thus we use $\mathcal{R}_{1}(\mathbf{B})=\|\mathbf{B}\|_{*}$ to encourage low-rankness. A widely used regularizer to encourage entry-wise sparsity is $\ell_{1}$ norm, such as Lasso in classical linear regression (Tibshirani, 1996). We use $\mathcal{R}_{2}(\mathbf{B})=\|\mathbf{B}\|_{1}:=\sum_{j=1}^{d_{1}} \sum_{k=1}^{d_{2}}\left|\mathbf{B}_{j k}\right|$ as the sparsity regularizer.

The convex optimization problem 2.2 includes two regularizers and the optimization problem with one penalty can be solved using a proximal gradient algorithm. Thus we can use the incremental proximal gradient method Bertsekas, 2011). Specifically, denoting $\ell(\mathbf{B})=\frac{1}{n} \sum_{i=1}^{n} \rho_{\tau}\left(y_{i}-\right.$ $\left.\left\langle\mathbf{B}, \mathbf{Z}_{i}\right\rangle\right)$, the incremental proximal gradient method operates on $\mathcal{R}_{1}$ and $\mathcal{R}_{2}$ in turn, and treats $\ell(\mathbf{B})$ in a (sub-)gradient step. The $t$ th iteration of the 
algorithm computes

$$
\begin{aligned}
& \mathbf{B}_{1}^{t}=\arg \min \left\{\mathcal{R}_{1}(\mathbf{B})+\frac{1}{2 \gamma}\left\|\mathbf{B}-\mathbf{B}^{t-1}\right\|_{F}^{2}\right\}, \\
& \mathbf{B}_{2}^{t}=\arg \min \left\{\mathcal{R}_{2}(\mathbf{B})+\frac{1}{2 \gamma}\left\|\mathbf{B}-\mathbf{B}_{1}^{t}\right\|_{F}^{2}\right\}, \\
& \mathbf{B}^{t}=\mathbf{B}_{2}^{t}-\gamma \nabla \ell\left(\mathbf{B}_{2}^{t}\right),
\end{aligned}
$$

where $\nabla \ell(\mathbf{B})$ is a sub-derivative of the loss, and $\gamma$ is the step size. The pseudo-code is presented in Algorithm 1. The initial value $\mathbf{B}^{0}$ is a matrix with independent standard normal entries. In fact, since the optimization problem is convex, initial estimator would have little effect in our procedure. For the step size, setting $\gamma$ to be too large may make the algorithm fail to converge, while a too small value would make the convergence very slow. In our simulations, after some exploration, the step size $\gamma$ is set to 0.1, which is satisfactory in our numerical studies. Investigation of a more principled and adaptive approach for step size will be left for future study. We stop the algorithm when the decrease of objective function value is less than $10^{-5}$. Because the algorithm can be seen as a special case of the incremental proximal gradient method, its numerical convergence is guaranteed by Proposition 3 and Proposition 4 of Bertsekas (2011). 
$\overline{\text { Algorithm } 1 \text { Incremental proximal gradient method for quantile matrix }}$ regression.

Input: Initial value $\mathbf{B}^{0}, \gamma$

repeat

$\operatorname{SVD}$ for $\mathbf{B}^{t-1}: \mathbf{B}^{t-1}=\mathbf{U} \operatorname{diag}\left(\sigma_{1}, \ldots, \sigma_{\min \left\{d_{1}, d_{2}\right\}}\right) \mathbf{V}^{\top}$

$\tilde{\sigma}_{j}=\operatorname{sign}\left(\sigma_{j}\right)\left(\left|\sigma_{j}\right|-\gamma \lambda_{1}\right)_{+}$, for $j=1, \ldots, \min \left\{d_{1}, d_{2}\right\}$

$\mathbf{B}_{1}^{t}=\mathbf{U} \operatorname{diag}\left(\tilde{\sigma}_{1}, \ldots, \tilde{\sigma}_{\min \left\{d_{1}, d_{2}\right\}}\right) \mathbf{V}^{\top}$

$\left(\mathbf{B}_{2}^{t}\right)_{j k}=\operatorname{sign}\left(\left(\mathbf{B}_{1}^{t}\right)_{j k}\right)\left(\left|\left(\mathbf{B}_{1}^{t}\right)_{j k}\right|-\gamma \lambda_{2}\right)_{+}$, for $j=1, \ldots, d_{1}, k=1, \ldots, d_{2}$

$\mathbf{B}^{t}=\mathbf{B}_{2}^{t}-\gamma \nabla \ell\left(\mathbf{B}_{2}^{t}\right)$

until convergence criterion is met

\subsection{Application to quadratic linear regression}

We consider the regression model with interaction effects

$$
Q_{\tau}(y \mid \mathbf{x})=\xi_{0}+\sum_{j=1}^{p} \xi_{j} x_{j}+\sum_{j, k=1}^{p} \beta_{j k} x_{j} x_{k}
$$

where $\mathbf{x}=\left(x_{1}, \ldots, x_{p}\right)^{\top}$ is the $p$-dimensional covariate, $\xi_{0}$ is the intercept, $\boldsymbol{\xi}=\left(\xi_{1}, \ldots, \xi_{p}\right)$ and $\boldsymbol{\beta}=\left(\beta_{11}, \ldots, \beta_{p p}\right)$ are the main effects and the interaction effects, respectively. For identifiability, we assume $\beta_{j k}=\beta_{k j}$. Model 2.3 can be reformulated into a matrix regression form by rearranging the coefficients into a matrix $\mathbf{B} \in \mathbb{R}^{(p+1) \times(p+1)}$, with $\mathbf{B}_{0,0}=\xi_{0}$, $\mathbf{B}_{j, 0}=\mathbf{B}_{0, j}=\xi_{j} / 2$ and $\mathbf{B}_{j, k}=\beta_{j k}$. In this way, model 2.3 becomes 2.1 with $\mathbf{Z}=\left(1, x_{1}, x_{2}, \ldots, x_{p}\right)^{\top}\left(1, x_{1}, x_{2}, \ldots, x_{p}\right)$. Dimension reduction in tra- 
ditional interaction effects models often only consider the sparsity structure. The advantage of writing the model into matrix form is that we can not only impose sparsity assumption, but also low-rankness assumption to further reduce dimension, which is useful when the number of nonzero entries is still large.

Since $\mathbf{B}$ is a symmetric matrix, the estimate $\widehat{\mathbf{B}}$ should be the minimizer of objective function 2.2 in the set of symmetric matrices in $\mathbb{R}^{(p+1) \times(p+1)}$. The incremental proximal gradient method can also deal with the case easily by changing the original gradient step by $\mathbf{B}^{t}=P_{\boldsymbol{S}^{p+1}}\left(\mathbf{B}_{2}^{t}-\gamma \nabla \ell\left(\mathbf{B}_{2}^{t}\right)\right)$, where $\boldsymbol{S}^{p+1}$ is the set of symmetric matrices in $\mathbb{R}^{(p+1) \times(p+1)}$ and $P_{\boldsymbol{S}^{p+1}}$ denotes the projection on $\boldsymbol{S}^{p+1}$. This can be written more explicitly as $\mathbf{B}^{t}=\frac{1}{2}\left[\left(\mathbf{B}_{2}^{t}-\gamma \nabla \ell\left(\mathbf{B}_{2}^{t}\right)\right)^{\top}+\left(\mathbf{B}_{2}^{t}-\gamma \nabla \ell\left(\mathbf{B}_{2}^{t}\right)\right)\right]$. However, it is easy to see that as long as the initial value of $\mathbf{B}$ is symmetric, all subsequent steps still produce a symmetric matrix and the projection step is redundant.

\section{Theoretical properties}

In this section, we establish an upper bound for the estimation error of $\widehat{\mathbf{B}}$ obtained by 2.2 in the high-dimensional scenario. There are two key elements that allow us to derive the upper bound, following the pioneering work of Negahban et al. (2012). The first is the concept of decomposability 
for penalties. For a subspace $\mathbb{M} \subset \mathbb{R}^{d_{1} \times d_{2}}$, define its orthogonal complement as

$$
\mathbb{M}^{\perp}=\left\{\mathbf{V} \in \mathbb{R}^{d_{1} \times d_{2}} ;\langle\mathbf{U}, \mathbf{V}\rangle=0 \text { for all } \mathbf{U} \in \mathbb{M}\right\}
$$

Given a pair of subspaces $\mathbb{M} \subseteq \overline{\mathbb{M}} \subset \mathbb{R}^{d_{1} \times d_{2}}$, a regularizer $\mathcal{R}$ is decomposable with respect to $\left(\mathbb{M}, \bar{M}^{\perp}\right)$ if

$$
\mathcal{R}(\mathbf{U}+\mathbf{V})=\mathcal{R}(\mathbf{U})+\mathcal{R}(\mathbf{V}), \text { for all } \mathbf{U} \in \mathbb{M} \text { and } \mathbf{V} \in \overline{\mathbb{M}}^{\perp}
$$

When $\mathbf{B}$ is a rank- $r$ matrix with $r \leq \min \left\{d_{1}, d_{2}\right\}$, let $\mathbb{U} \subseteq \mathbb{R}^{d_{1}}$ and $\mathbb{V} \subseteq \mathbb{R}^{d_{2}}$ be a pair of $r$-dimensional subspaces spanned by the left and the right singular vectors of $\mathbf{B}$, respectively. Consider subspaces

$$
\begin{aligned}
& \mathbb{M}_{1}=\left\{\mathbf{A} \in \mathbb{R}^{d_{1} \times d_{2}} \mid \operatorname{row}(\mathbf{A}) \subseteq \mathbb{V}, \operatorname{col}(\mathbf{A}) \subseteq \mathbb{U}\right\} \\
& \overline{\mathbb{M}}_{1}^{\perp}=\left\{\mathbf{A} \in \mathbb{R}^{d_{1} \times d_{2}} \mid \operatorname{row}(\mathbf{A}) \subseteq \mathbb{V}^{\perp}, \operatorname{col}(\mathbf{A}) \subseteq \mathbb{U}^{\perp}\right\},
\end{aligned}
$$

where $\operatorname{row}(\mathbf{A})$ and $\operatorname{col}(\mathbf{A})$ are the row and the column space for the matrix A. It is known that $\mathcal{R}_{1}$ is decomposable with respect to $\left(\mathbb{M}_{1}, \overline{\mathbb{M}}_{1}^{\perp}\right)$. For the sparsity penalty $\mathcal{R}_{2}$, let $\mathcal{S} \subseteq\left\{1, \ldots, d_{1}\right\} \times\left\{1, \ldots, d_{2}\right\}$ be the indices of nonzero entries with cardinality $|\mathcal{S}|=s$, and let $\mathcal{S}^{\perp}=\left\{1, \ldots, d_{1}\right\} \times$ $\left\{1, \ldots, d_{2}\right\} \backslash \mathcal{S}$. Then $\mathcal{R}_{2}$ is decomposable with respect to $\left(\mathbb{M}_{2}, \overline{\mathbb{M}}_{2}^{\perp}\right)$ where

$$
\begin{aligned}
& \mathbb{M}_{2}=\bar{M}_{2}=\left\{\mathbf{A} \in \mathbb{R}^{d_{1} \times d_{2}} \mid \mathbf{A}_{i j}=0 \text { for all }(i, j) \in \mathcal{S}^{\perp}\right\} \\
& \overline{\mathbb{M}}_{2}^{\perp}=\left\{\mathbf{A} \in \mathbb{R}^{d_{1} \times d_{2}} \mid \mathbf{A}_{i j}=0 \text { for all }(i, j) \in \mathcal{S}\right\} .
\end{aligned}
$$


The second property concerns the restricted set that $\widehat{\mathbf{B}}-\mathbf{B}$ can be proved to be in. Let $\mathbf{P}_{\mathbf{U}^{\perp}}$ and $\mathbf{P}_{\mathbf{V}^{\perp}}$ be the projection matrices to spaces $\mathbb{U}^{\perp}$ and $\mathbb{V}^{\perp}$, respectively. Then for a matrix $\boldsymbol{\Delta}$, define $\boldsymbol{\Delta}^{\prime \prime}=\mathbf{P}_{\mathbf{U}^{\perp}} \boldsymbol{\Delta} \mathbf{P}_{\mathbf{V}^{\perp}} \in \bar{M}_{1}^{\perp}$ (this is actually the projection of $\boldsymbol{\Delta}$ on $\overline{\mathbb{M}}_{1}^{\perp}$ ) and $\boldsymbol{\Delta}^{\prime}=\boldsymbol{\Delta}-\boldsymbol{\Delta}^{\prime \prime} \in \overline{\mathrm{M}}_{1}$. Besides, we denote by $\boldsymbol{\Delta}_{\mathcal{S}}$ the matrix in which $\left(\boldsymbol{\Delta}_{\mathcal{S}}\right)_{i j}=\boldsymbol{\Delta}_{i j}$ if $(i, j) \in \mathcal{S}$, and $\left(\boldsymbol{\Delta}_{\mathcal{S}}\right)_{i j}=0$ if $(i, j) \notin \mathcal{S}\left(\boldsymbol{\Delta}_{\mathcal{S}}\right.$ is the projection of $\boldsymbol{\Delta}$ on $\left.\mathbb{M}_{2}\right)$. Then the restricted set in our setting is defined as

$$
\mathbb{C}=\left\{\boldsymbol{\Delta} \mid \lambda_{1} \mathcal{R}_{1}\left(\boldsymbol{\Delta}^{\prime \prime}\right)+\lambda_{2} \mathcal{R}_{2}\left(\boldsymbol{\Delta}_{\mathcal{S}^{\perp}}\right) \leq 3 \lambda_{1} \mathcal{R}_{1}\left(\boldsymbol{\Delta}^{\prime}\right)+3 \lambda_{2} \mathcal{R}_{2}\left(\boldsymbol{\Delta}_{\mathcal{S}}\right)\right\}
$$

The value 3 in the above is somewhat arbitrary and can be replaced by any constant larger than 1 . For convenience in theoretical analysis, in the following we will write $\lambda_{1}=\lambda \alpha, \lambda_{2}=\lambda(1-\alpha)$, with $\lambda=\lambda_{1}+\lambda_{2}$ and $\alpha=\lambda_{1} / \lambda$. Then the restricted set can also be written as

$$
\alpha \mathcal{R}_{1}\left(\boldsymbol{\Delta}^{\prime \prime}\right)+(1-\alpha) \mathcal{R}_{2}\left(\boldsymbol{\Delta}_{\mathcal{S}^{\perp}}\right) \leq 3\left(\alpha \mathcal{R}_{1}\left(\boldsymbol{\Delta}^{\prime}\right)+(1-\alpha) \mathcal{R}_{2}\left(\boldsymbol{\Delta}_{\mathcal{S}}\right)\right)
$$

Let $p=d_{1} d_{2}, \mathbf{z}_{i}=\operatorname{vec}\left(\mathbf{Z}_{i}\right)$. In order to obtain the upper bound, we assume the following conditions. In the following, $C$ denotes a generic positive constant whose value can change at different appearances.

C1. $\mathbf{J}:=E\left[\mathbf{z}_{i} \mathbf{z}_{i}^{\top}\right]$ is positive definite with its maximum eigenvalue $\sigma_{\max }(\mathbf{J})$ bounded by a constant. 
C2. $\mathbf{z}_{i}=\operatorname{vec}\left(\mathbf{Z}_{i}\right)$ is sub-Gaussian in the sense that there exists a constant $C>0$, for any unit norm vector a, we have $E\left[e^{t \mathbf{a}^{\top} \mathbf{z}_{i}}\right] \leq e^{C t^{2}}, \forall t>0$.

C3. With $\mathbf{B}$ denoting the true coefficient matrix, there is a constant $c_{1}>0$ such that $E\left[\rho_{\tau}\left(y_{i}-\left\langle\mathbf{B}+\boldsymbol{\Delta}, \mathbf{Z}_{i}\right\rangle\right)\right]-E\left[\rho_{\tau}\left(y_{i}-\left\langle\mathbf{B}, \mathbf{Z}_{i}\right\rangle\right)\right] \geq c_{1}\left(\|\boldsymbol{\Delta}\|_{F}^{2} \wedge\right.$ $\left.\|\Delta\|_{F}\right)$, for all $\boldsymbol{\Delta} \in \mathbb{C}$, where $\|.\|_{F}$ denotes the Frobenius norm.

Condition $\mathrm{C} 1$ is a mild moment assumption. Sub-Gaussianity of $\mathbf{Z}_{i}$ is required to bound different norms of a single fixed random matrix as in Lemma 2 in the supplement material, and such a light-tail condition is often used in high-dimensional asymptotic analysis. Finally, C3 can be satisfied using more primitive assumptions including the boundedness conditions for conditional density of $y_{i}$ given $\mathbf{Z}_{i}$ and inf $\boldsymbol{\Delta} \in \mathbb{C}_{\mathbb{C}} \frac{\left(E\left|\left\langle\boldsymbol{\Delta}, \mathbf{Z}_{i}\right\rangle\right|^{2}\right)^{\frac{3}{2}}}{E\left|\left\langle\boldsymbol{\Delta}, \mathbf{Z}_{i}\right\rangle\right|^{3}}>0$. And the latter can be satisfied when, for example, $\mathbf{Z}_{i}$ is Gaussian. The proof is similar to that of Lemma 4 (3.7) of Belloni and Chernozhukov (2011), we show this in the supplementary material.

Theorem 1. Suppose the true parameter $\mathbf{B}$ has rank $r$ and $s$ nonzero entries, and assumptions C1-C3 hold. If $\alpha \in[0,1]$ and $\lambda \geq C \min \left\{\sqrt{\frac{d_{1}+d_{2}}{n \alpha^{2}}}, \sqrt{\frac{\log _{p}}{n(1-\alpha)^{2}}}\right\}$ for a sufficiently large $C>0$, with probability approaching one, we have

$$
\|\widehat{\mathbf{B}}-\mathbf{B}\|_{F} \leq C \lambda(\alpha \sqrt{r}+(1-\alpha) \sqrt{s})
$$

as long as the right-hand size above is o(1). In particular, taking $\lambda \asymp$ 


$$
\begin{aligned}
& C \min \left\{\sqrt{\frac{d_{1}+d_{2}}{n \alpha^{2}}}, \sqrt{\frac{\log p}{n(1-\alpha)^{2}}}\right\}, \\
& \|\widehat{\mathbf{B}}-\mathbf{B}\|_{F} \leq C \min \left\{\sqrt{\frac{\left(d_{1}+d_{2}\right) r}{n}}+\frac{1-\alpha}{\alpha} \sqrt{\frac{\operatorname{slog} p}{n}}, \frac{\alpha}{1-\alpha} \sqrt{\frac{\left(d_{1}+d_{2}\right) r}{n}}+\sqrt{\frac{\operatorname{slog} p}{n}}\right\} .
\end{aligned}
$$

Note that we allow $d_{1}$ and $d_{2}$ (and so does $p=d_{1} d_{2}$ ) to diverge with $n$. On the other hand, the growth rate of $d_{1}, d_{2}$ and $s$ has to satisfy $\lambda(\alpha \sqrt{r}+(1-\alpha) \sqrt{s})=o(1)$. The theorem shows that the estimator can track the better performer of the nuclear-norm penalized estimator and the sparse (lasso) estimator. When $\alpha$ is sufficiently close to 1 , the rate becomes $\sqrt{\left(d_{1}+d_{2}\right) r / n}$ which is the same as the rate in Negahban and Wainwright (2011) for least squares regression. On the other hand, when $\alpha \approx 0$, the rate becomes $\sqrt{s \log p / n}$ as in Belloni and Chernozhukov (2011).

Remark 1. Theorem 1 established error bound for a single quantile level $\tau \in(0,1)$. Suppose now model (2.1) is true for $\tau \in\left[\tau_{L}, \tau_{U}\right] \subset(0,1)$. When considering the uniform error bound for $\tau \in\left[\tau_{L}, \tau_{U}\right]$, an additional condition on the true coefficient matrix $\mathbf{B}(\tau)$ is needed. That is, there exists a (diverging) constant $L>0$ such that

$$
\left\|\mathbf{B}(\tau)-\mathbf{B}\left(\tau^{\prime}\right)\right\|_{F} \leq L\left|\tau-\tau^{\prime}\right|, \text { for all } \tau, \tau^{\prime} \in\left[\tau_{L}, \tau_{U}\right]
$$

Then, if we make assumption C3 also uniform over $\tau$, by following the same proof strategy as in Belloni et al. (2019), we expect that one can establish 
the same bound uniformly over $\tau \in\left[\tau_{L}, \tau_{U}\right]$. However, we leave the details out and focus on the single $\tau$ case here.

Remark 2. When $\alpha=0$, the rate is only near oracle. We think that employing the adaptive Lasso penalty $\sum_{j, k=1} w_{j k}\left|\mathbf{B}_{j, k}\right|$, where $w_{j k}=\frac{1}{\left|\hat{\mathbf{B}}_{j, k}\right|}$ and the initial estimator $\tilde{\mathbf{B}}$ is obtained by Lasso penalized quantile regression, would lead to the oracle rate, under additional conditions which involves a signal strength requirement, that is a lower bound on $\inf _{(j, k) \in S}\left|\mathbf{B}_{j, k}\right|$. Signal strength conditions can be restrictive but it is usually required for oracle property, see for example Zhao and Yu (2006); Meinshausen and Bühlmann (2006); Bühlmann and van de Geer (2011); Zheng et al. (2015); Ndaoud (2019). A nonconvex penalty can also possibly achieve oracle rate under such conditions. It would be interesting to establish oracle rate for quantile matrix regression with both sparsity and low-rankness constraint, which requires further work.

\section{Numerical results}

We consider the quadratic quantile regression problem. The response is obtained by $y_{i}=\left\langle\mathbf{B}, \mathbf{Z}_{i}\right\rangle+\varepsilon_{i}$, where $\mathbf{Z}_{i}=\left(1, x_{i 1}, \ldots, x_{i p}\right)^{\top}\left(1, x_{i 1}, \ldots, x_{i p}\right)$ with $x_{i j}$ generated independently from a standard normal distribution, and the random error is generated by $\varepsilon_{i}=\left(1+0.2\left|x_{i, 1}\right|\right) \epsilon_{i}$ with $\epsilon_{i} \sim N\left(-q_{\tau}, \sigma^{2}\right)$ 
and $q_{\tau}$ is the $\tau$ th quantile of the Gaussian distribution $N\left(0, \sigma^{2}\right)$. The coefficient $\mathbf{B}$ is a rank- $r$ symmetric matrix which is obtained by $\mathbf{U D U}^{\top}$, where $\mathbf{U} \in \mathbb{R}^{(p+1) \times r}$ is the top $r$ left singular vectors of a matrix with independent standard normal entries. In order to generate a sparse matrix $\mathbf{B}$, we first generate $\mathbf{U} \in \mathbb{R}^{p^{\prime} \times r}$ and then insert $p+1-p^{\prime}$ zero rows in $\mathbf{U}$. Let $q=p^{\prime} /(p+1)$ be the proportion of zero rows in $\mathbf{U}$ and we investigate the effect of different values of $q$ in our simulations.

We applied the proposed method to estimate the coefficient matrix B. First, we take sample size $n=300,500$ and 700, and the dimension is set to be $p=30$. The true rank $r$ is 3 and we set $q=0.5$ and $\sigma=3$. The tuning parameters $\lambda_{1}$ and $\lambda_{2}$ are selected by five-fold cross-validation, and the step size $\gamma$ is always set to be 0.1 . We use $\|\widehat{\mathbf{B}}-\mathbf{B}\|_{F}$ as errors reported in the simulation results. All simulations are repeated 200 times. Figure 1 compares our method with the Lasso approach for the model with interactions. We see the errors decrease with $n$ and our approach performs better than Lasso as expected.

In the results reported in Figure 2 , we set $n=500, q=0.3$, and vary the dimension $p \in\{30,50,70\}$. In Figure 3 we report the results with $n=500, p=30$ and varying $q \in\{0.3,0.5,0.7,0.9,1\}$ (corresponding to about $8 \%, 23 \%, 46 \%, 76 \%$ and $100 \%$ nonzero entries). It can be seen that 
our approach outperforms Lasso in all cases, and the improvement is larger when $\mathbf{B}$ is denser.
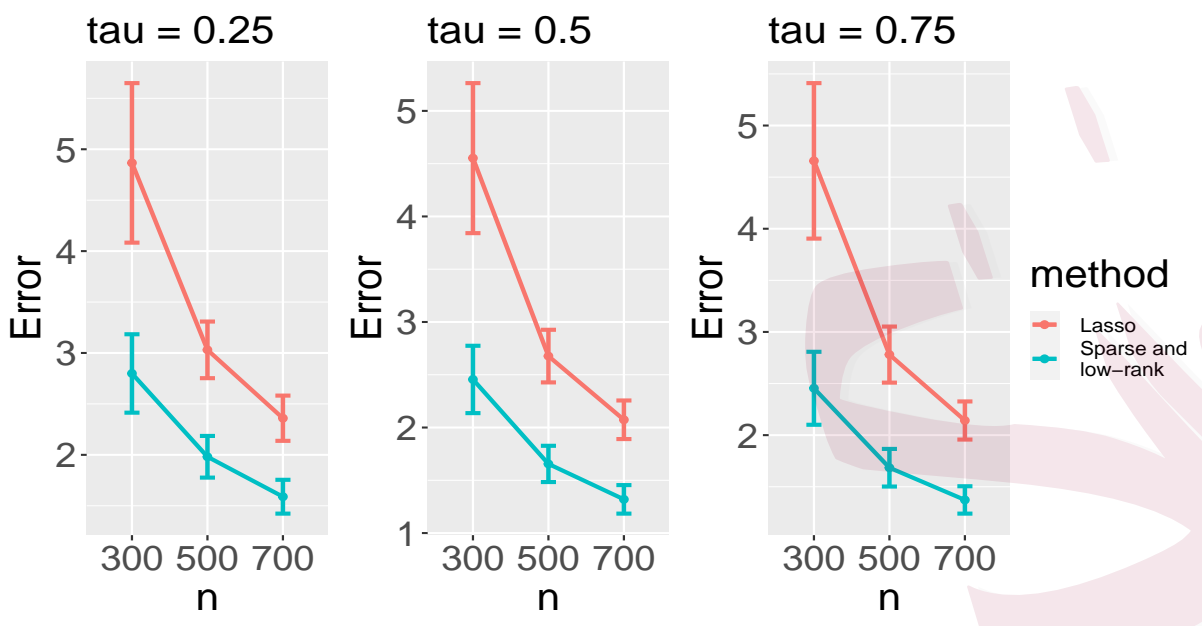

Figure 1: Estimation errors at quantile levels $\tau=0.25,0.5$ and 0.75 when $p=30, r=3, q=0.5$ and $\sigma=3$. The error bars represent \pm one standard deviation.

Moreover, we compare the proposed quantile regression approach at $\tau=$ 0.5 with the low-rank matrix mean regression Negahban and Wainwright, 2011). For both mean and 0.5 quantile regression, we use $\|\mathbf{B}\|_{*},\|\mathbf{B}\|_{1}$ or $\alpha\|\mathbf{B}\|_{*}+(1-\alpha)\|\mathbf{B}\|_{1}$ as regularizers. We take $n=500, r=3, q=$ $0.3, p=30,50$ and 70 , and the random error is generated from $N(0,1)$ and $t(3)$. The results reported in Table 1 show that the performance of mean regression may be better than median regression when random errors 

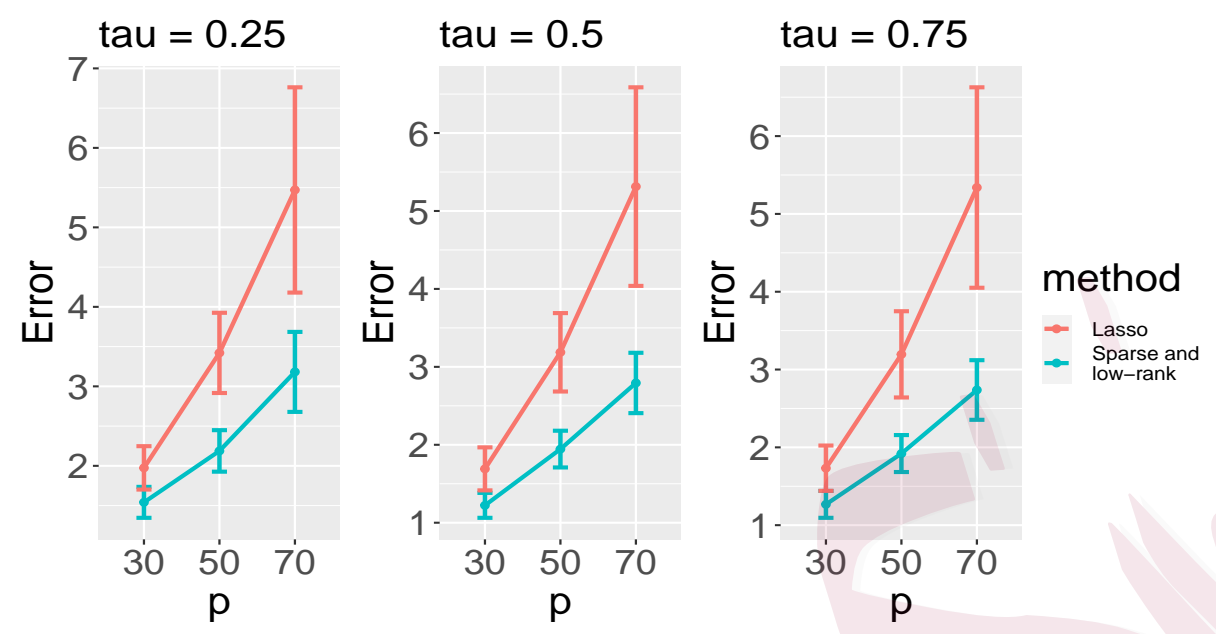

Figure 2: Estimation errors at quantile levels $\tau=0.25,0.5$ and 0.75 when $n=500, r=3, q=0.3$ and $\sigma=3$. The error bars represent \pm one standard deviation.

follow standard normal distribution (but not always so probably because we have heterogeneous errors here), but median regression outperforms mean regression with heavy-tailed errors. Besides, the computing time of different methods is reported in Table 2. The settings are the same as those in simulations.

Finally, we apply quadratic regression to 9 regression problems from the UCI machine learning repository. For each problem, we compare the proposed estimator with lasso estimator (with interaction effects). The test errors are obtained by cross-validation and the tuning parameters are 


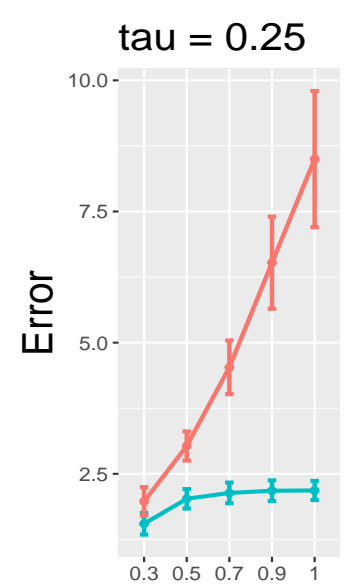

q

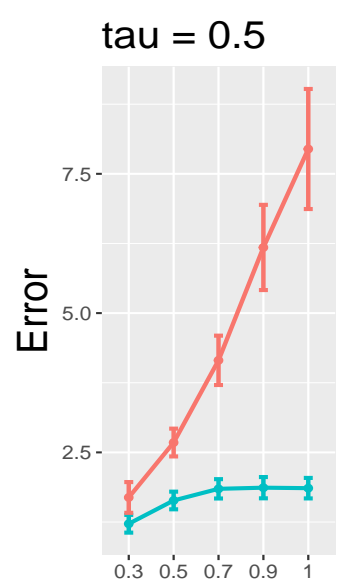

q

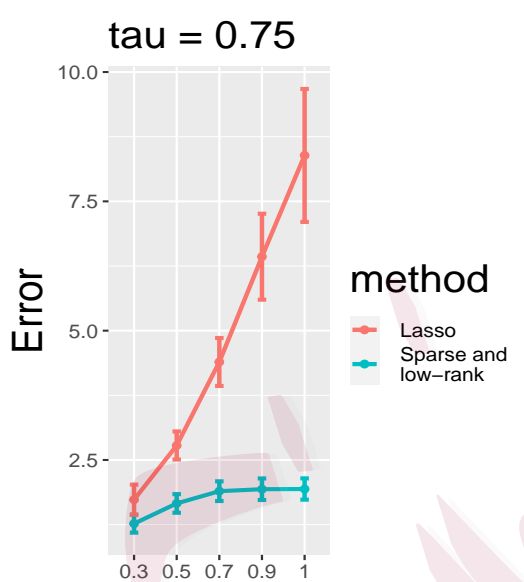

q

Figure 3: Estimation errors at quantile levels $\tau=0.25,0.5$ and 0.75 when $n=500, p=30, r=3, \sigma=3$ and $q$ ranges from 0.3 to 1 . The error bars represent \pm one standard deviation.

chosen again by 5 -fold cross-validation on the training set. The results are shown in Table 3. It can be seen that introducing the low-rank regularizer improves the performance.

\section{Conclusion and discussion}

In this paper, we proposed a convex regularized optimization approach for quantile regression with matrix covariates. The motivation of our work is the wide application of matrix regression and the lack of studies in matrix quantile regression. In order to reduce the effective number of parame- 
Table 1: Estimation error for the proposed method (sparse and low-rank) at quantile level $\tau=0.5$, and mean regression (least square), when $n=500$, $r=3, q=0.3$ and $p=30,50$ and 70 . Numbers in the parentheses denote the standard errors.

\begin{tabular}{|c|c|c|c|c|c|}
\hline & Regularizer & Method & $p=30$ & $p=50$ & $p=70$ \\
\hline \multirow{6}{*}{$N(0,1)$} & \multirow{2}{*}{$\|\mathbf{B}\|_{*}$} & 0.5 quantile & $0.60(0.08)$ & $1.21(0.19)$ & $2.72(0.61)$ \\
\hline & & Mean & $0.62(0.09)$ & $1.81(0.38)$ & $3.28(0.68)$ \\
\hline & \multirow{2}{*}{$\|\mathbf{B}\|_{1}$} & 0.5 quantile & $0.58(0.10)$ & $1.81(0.47)$ & $4.83(1.45)$ \\
\hline & & mean & $0.52(0.09)$ & $2.37(0.62)$ & $4.85(1.33)$ \\
\hline & \multirow{2}{*}{$\|\mathbf{B}\|_{*}$ and $\|\mathbf{B}\|_{1}$} & 0.5 quantile & $0.43(0.06)$ & $0.82(0.10)$ & $1.64(0.25)$ \\
\hline & & mean & $0.38(0.05)$ & $0.74(0.14)$ & $1.55(0.34)$ \\
\hline \multirow{6}{*}{$t(3)$} & \multirow{2}{*}{$\|\mathbf{B}\|_{*}$} & 0.5 quantile & $0.75(0.11)$ & $1.49(0.23)$ & $3.03(0.66)$ \\
\hline & & Mean & $0.88(0.16)$ & $2.02(0.38)$ & $3.40(0.70)$ \\
\hline & \multirow{2}{*}{$\|\mathbf{B}\|_{1}$} & 0.5 quantile & $0.73(0.13)$ & $2.19(0.58)$ & $5.07(1.46)$ \\
\hline & & mean & $0.88(0.21)$ & $2.59(0.62)$ & $4.98(1.32)$ \\
\hline & \multirow{2}{*}{$\|\mathbf{B}\|_{*}$ and $\|\mathbf{B}\|_{1}$} & 0.5 quantile & $0.52(0.07)$ & $1.00(0.13)$ & $1.88(0.32)$ \\
\hline & & mean & $0.60(0.12)$ & $1.07(0.20)$ & $1.90(0.42)$ \\
\hline
\end{tabular}


Table 2: Averaging computing time (in seconds) of the proposed sparse and low-rank method, Lasso, two-stage and least square approaches to complete the simulations, using $\mathrm{R}$ (version 3.6.3) on our desktop computer with 3.40GHz CPU.

\begin{tabular}{|c|c|c|c|}
\hline & $p=30$ & $p=50$ & $p=70$ \\
\hline Sparse and low-rank & 68.71 & 144.36 & 322.69 \\
\hline Lasso & 24.58 & 36.81 & 113.46 \\
\hline Least square & 86.38 & 168.65 & 236.29 \\
\hline
\end{tabular}

ters in the high-dimensional setting, two regularizers corresponding to lowrankness and sparsity are imposed at the same time. We establish the upper bound on the estimation error of the proposed estimator and develop an algorithm based on incremental proximal gradient. We apply the proposed method to quadratic quantile regression, where the covariates and their interactions can be reformed into a matrix. The advantage of the proposed method in quadratic regression problems is demonstrated by simulations and real data analysis.

When studying quadratic regression, the hierarchy restriction that an interaction can only be included in the model if both or either main effects are selected, are often assumed, see for example Bien et al. (2013); Hao and 
Table 3: Test errors for 9 regression problems at quantile levels $\tau=0.25$, 0.5 and 0.75 .

\begin{tabular}{|c|c|c|c|c|c|}
\hline dataset & $n$ & $p$ & $\tau$ & Lasso & Sparse \& low-rank \\
\hline \multirow{3}{*}{$\begin{array}{l}\text { Wisconsin } \\
\text { Prognostic Breast } \\
\text { Cancer }\end{array}$} & \multirow{3}{*}{155} & \multirow{3}{*}{32} & 0.25 & 1.46 & 0.93 \\
\hline & & & 0.5 & 2.54 & 1.22 \\
\hline & & & 0.75 & 1.89 & 0.81 \\
\hline \multirow{3}{*}{$\begin{array}{l}\text { Residential } \\
\text { Building-Sales Price }\end{array}$} & \multirow{3}{*}{298} & \multirow{3}{*}{26} & 0.25 & 0.18 & 0.11 \\
\hline & & & 0.5 & 0.21 & 0.09 \\
\hline & & & 0.75 & 0.08 & 0.07 \\
\hline \multirow{3}{*}{$\begin{array}{l}\text { Residential } \\
\text { Building-- } \\
\text { Construction }\end{array}$} & \multirow{3}{*}{298} & \multirow{3}{*}{26} & 0.25 & 0.21 & 0.14 \\
\hline & & & 0.5 & 0.10 & 0.06 \\
\hline & & & 0.75 & 0.07 & 0.04 \\
\hline \multirow{3}{*}{$\begin{array}{l}\text { Real Estate } \\
\text { Valuation }\end{array}$} & \multirow{3}{*}{331} & \multirow{3}{*}{6} & 0.25 & 2.34 & 2.03 \\
\hline & & & 0.5 & 2.85 & 2.72 \\
\hline & & & 0.75 & 2.55 & 2.37 \\
\hline \multirow{3}{*}{ Forest Fires } & \multirow{3}{*}{414} & \multirow{3}{*}{10} & 0.25 & 0.28 & 0.28 \\
\hline & & & 0.5 & 0.58 & 0.53 \\
\hline & & & 0.75 & 0.54 & 0.52 \\
\hline \multirow{3}{*}{$\begin{array}{l}\text { Geographical } \\
\text { Original of Music-- } \\
\text { Latitude }\end{array}$} & \multirow{3}{*}{847} & \multirow{3}{*}{68} & 0.25 & 1.91 & 0.97 \\
\hline & & & 0.5 & 0.68 & 0.56 \\
\hline & & & 0.75 & 1.23 & 0.44 \\
\hline \multirow{3}{*}{$\begin{array}{l}\text { Geographical } \\
\text { Original of Music-- } \\
\text { Longitude }\end{array}$} & \multirow{3}{*}{847} & \multirow{3}{*}{68} & 0.25 & 2.72 & 1.47 \\
\hline & & & 0.5 & 1.31 & 1.14 \\
\hline & & & 0.75 & 1.23 & 0.98 \\
\hline \multirow{3}{*}{$\begin{array}{l}\text { PM2.5 Beijing-- } \\
\text { Aotizhongxin }\end{array}$} & \multirow{3}{*}{1071} & & 0.25 & 0.12 & 0.10 \\
\hline & & 11 & 0.5 & 0.12 & 0.10 \\
\hline & & & 0.75 & 0.09 & 0.08 \\
\hline \multirow{3}{*}{ Wine Quality-Red } & \multirow{3}{*}{1279} & \multirow{3}{*}{11} & 0.25 & 0.20 & 0.19 \\
\hline & & & 0.5 & 0.26 & 0.24 \\
\hline & & & 0.75 & 0.22 & 0.21 \\
\hline
\end{tabular}

Zhang (2014). When using entry-wise Lasso as sparsity regularizer, hierar-

chical structure is not incorporated. Strong heredity (an interaction effect can be selected only if both main effects are selected) could be incorporated by replacing $\|\mathbf{B}\|_{1}$ by a hierarchical penalty, for example, the composite 
absolute penalty (Zhao et al., 2009)

$$
\mathcal{R}_{2}(\mathbf{B})=\sum_{j, k=1}^{p}\left(\left|\mathbf{B}_{j, k}\right|+\left\|\left(\mathbf{B}_{j, 0}, \mathbf{B}_{0, k}, \mathbf{B}_{j, k}\right)\right\|_{2}\right)
$$

The theoretical guarantees for this hierarchical penalty require further study. 


\section{Supplementary Materials}

Proof of the theorem are contained in the Supplementary Materials.

\section{Acknowledgements}

We sincerely thank the Editor, Associate Editor, and two anonymous reviewers for their insightful comments that have greatly improved the manuscript.

The research of Zhu is supported by National Natural Science Foundation of China (11731011, 11690013, 12071087). The research of Heng Lian is supported by Project 11871411 from NSFC and CityU Shenzhen Research Institute, and by Hong Kong General Research Fund 11301718, 11300519 and 11300721.

\section{References}

Agarwal, A., Negahban, S., and Wainwright, M. J. "Noisy matrix decomposition via convex relaxation: Optimal rates in high dimensions." The Annals of Statistics, 40(2):1171-1197 (2012).

Argyriou, A., Evgeniou, T., and Pontil, M. "Convex multi-task feature learning." Machine learning, 73(3):243-272 (2008).

Argyriou, A., Micchelli, C. A., and Pontil, M. "On spectral learning." Journal of Machine Learning Research, 11(2):935-953 (2010). 
Belloni, A. and Chernozhukov, V. "l1 -penalized quantile regression in high-dimensional sparse models." The Annals of Statistics, 39(1):82-130 (2011).

Belloni, A., Chernozhukov, V., Chetverikov, D., and Fernández-Val, I. "Conditional quantile processes based on series or many regressors." Journal of Econometrics, 213(1):4-29 (2019)

Bertsekas, D. P. "Incremental proximal methods for large scale convex optimization." Mathematical programming, 129(2):163-195 (2011).

Bien, J., Taylor, J., and Tibshirani, R. "A lasso for hierarchical interactions." Annals of Statistics, 41(3):1111-1141 (2013).

Bühlmann, P. and van de Geer, S. Statistics for High-Dimensional data: Methods, Theory and Applications (2011).

Bunea, F., She, Y., and Wegkamp, M. H. "Optimal selection of reduced rank estimators of high-dimensional matrices." The Annals of Statistics, 39(2):1282-1309 (2011).

Bunea, F., She, Y., and Wegkamp, M. H. "Joint variable and rank selection for parsimonious estimation of high-dimensional matrices." The Annals of Statistics, 40(5):2359-2388 (2012).

Candes, E. J. and Plan, Y. "Matrix completion with noise." Proceedings of the IEEE, 98(6):925$936(2010)$.

Chao, S.-K., Volgushev, S., and Cheng, G. "Quantile processes for semi and nonparametric 
regression." Electronic Journal of Statistics, 11(2):3272-3331 (2017).

Chen, K., Chan, K.-S., and Stenseth, N. C. "Reduced rank stochastic regression with a sparse singular value decomposition." Journal of the Royal Statistical Society: Series B (Statistical Methodology), 74(2):203-221 (2012).

Choi, N. H., Li, W., and Zhu, J. "Variable selection with the strong heredity constraint and its oracle property." Journal of the American Statistical Association, 105(489):354-364 (2010).

Fan, J. and Li, R. "Variable selection via nonconcave penalized likelihood and its oracle properties." Journal of the American statistical Association, 96(456):1348-1360 (2001).

Fan, Y., Kong, Y., Li, D., and Zheng, Z. "Innovated interaction screening for high-dimensional nonlinear classification." The Annals of Statistics, 43(3):1243-1272 (2015).

Hao, N., Feng, Y., and Zhang, H. H. "Model selection for high-dimensional quadratic regression via regularization." Journal of the American Statistical Association, 113(522):615-625 (2018).

Hao, N. and Zhang, H. H. "Interaction screening for ultrahigh-dimensional data." Journal of the American Statistical Association, 109(507):1285-1301 (2014).

Kato, K. "Group Lasso for high dimensional sparse quantile regression models." arXiv preprint arXiv:1103.1458 (2011).

Koenker, R. Quantile Regression. New York: Cambridge University Press (2005). 
Koenker, R. and Bassett, G. "Regression quantiles." Econometrica, 46(1):33-50 (1978).

Koltchinskii, V. "Von Neumann entropy penalization and low-rank matrix estimation." The Annals of Statistics, 39(6):2936-2973 (2011).

Koltchinskii, V., Lounici, K., and Tsybakov, A. B. "Nuclear-norm penalization and optimal rates for noisy low-rank matrix completion." The Annals of Statistics, 39(5):2302-2329 (2011).

Ma, Z., Ma, Z., and Sun, T. "Adaptive estimation in two-way sparse reduced-rank regression." arXiv preprint arXiv:1403.1922 (2014).

Meinshausen, N. and Bühlmann, P. "High-dimensional graphs and variable selection with the lasso." The Annals of Statistics, 34(3):1436-1462 (2006).

Ndaoud, M. "Interplay of minimax estimation and minimax support recovery under sparsity." In Algorithmic Learning Theory, volume 98, 1-22. PMLR (2019).

Negahban, S. and Wainwright, M. J. "Estimation of (near) low-rank matrices with noise and high-dimensional scaling." The Annals of Statistics, 39:1069-1097 (2011).

Negahban, S. and Wainwright, M. J. "Restricted strong convexity and weighted matrix completion: Optimal bounds with noise." The Journal of Machine Learning Research, 13(1):16651697 (2012).

Negahban, S. N., Ravikumar, P., Wainwright, M. J., and Yu, B. "A unified framework for highdimensional analysis of $M$-estimators with decomposable regularizers." Statistical Science, 
27(4):538-557 (2012).

Pan, X. and Zhou, W.-X. "Multiplier bootstrap for quantile regression: non-asymptotic theory under random design." Information and Inference: A Journal of the IMA (2020).

Rohde, A. and Tsybakov, A. B. "Estimation of high-dimensional low-rank matrices." The Annals of Statistics, 39(2):887-930 (2011).

Tibshirani, R. "Regression shrinkage and selection via the lasso." Journal of the Royal Statistical Society. Series B (Methodological), 58(1):267-288 (1996).

Yi, C. and Huang, J. "Semismooth newton coordinate descent algorithm for elastic-net penalized huber loss regression and quantile regression." Journal of Computational and Graphical Statistics, 26(3):547-557 (2017).

Yu, L., Lin, N., and Wang, L. "A parallel algorithm for large-scale nonconvex penalized quantile regression." Journal of Computational and Graphical Statistics, 26(4):935-939 (2017).

Yuan, M., Ekici, A., Lu, Z., and Monteiro, R. "Dimension reduction and coefficient estimation in multivariate linear regression." Journal of the Royal Statistical Society: Series B (Statistical Methodology), 69(3):329-346 (2007).

Zhao, P., Rocha, G., and Yu, B. "The composite absolute penalties family for grouped and hierarchical variable selection." The Annals of Statistics, 37(6A):3468-3497 (2009).

Zhao, P. and Yu, B. "On model selection consistency of Lasso." The Journal of Machine Learning Research, 7:2541-2563 (2006). 
Zheng, Q., Peng, L., and He, X. "Globally adaptive quantile regression with ultra-high dimensional data." Annals of statistics, 43(5):2225 (2015).

Zhou, H. and Li, L. "Regularized matrix regression." Journal of the Royal Statistical Society: Series B (Statistical Methodology), 76(2):463-483 (2014).

Zou, H. and Hastie, T. "Regularization and variable selection via the elastic net." Journal of the Royal Statistical Society: Series B (Statistical Methodology), 67(2):301-320 (2005).

Department of Mathematics, City University of Hong Kong, Kowloon, Hong Kong

E-mail: wenqilu4-c@my.cityu.edu.hk

School of Management, Fudan University, Shanghai 200433, China

E-mail: zhuzy@fudan.edu.cn

Department of Mathematics, City University of Hong Kong, Kowloon, Hong Kong

E-mail: henglian@cityu.edu.hk 